\title{
Costly adult ADHD shunned by medical aids
}

Lack of clinical knowledge and financial support for adults with attention deficit hyperactive disorder (ADHD) are major barriers to treatment and diagnosis of a condition affecting an estimated one million South Africans aged 20 - 50 years.

This is the finding of a thesis completed by $\mathrm{Dr}$ Renata Schoeman, a psychiatrist and Stellenbosch University Business School (SBC) top-of-class MBA student. It involved a triangulated study: a retrospective database analysis of all claims submitted to one of the country's largest medical scheme administrators over a 2-year period, a survey of all registered psychiatrists (588, with 455 in private practice) treating adult ADHD patients, and a qualitative analysis of key local opinion leaders in the fields of psychiatry and neurology. She found that, left untreated or misdiagnosed, adult ADHD increased the risk of other psychiatric phenomena such as anxiety, mood disorder and substance abuse. At work, people with ADHD displayed poor time management, goal setting, stress management and organisational skills which had a major impact on colleagues. While medical schemes routinely covered childhood ADHD, they seldom provided benefits for adults, who struggled to afford private treatment over and above their monthly medical aid costs. This led to huge undertreatment. 'It's a costly, chronic disorder, with significant impact on the quality of life of patients and their families', she emphasises. She cites the condition's disability-adjusted life-years (DALYs) at 424/100 000 .

\section{Primary care clinical \\ knowledge poor}

GPs routinely misdiagnosed or inappropriately diagnosed complex presenting symptoms. They and many psychiatrists (especially those who qualified before adult ADHD was recognised as a disorder) had limited knowledge about adult ADHD. 'Also, you cannot assess properly in 15 minutes. It often presents with comorbidities. Those who primarily complain of ADHD symptoms are often students and professionals wanting the drugs for study purposes, so vigilance is needed. She described the longstanding student practice of misusing ADHD medication to improve concentration (without being diagnosed with the condition) as 'irresponsible and potentially risky'.

Schoeman has developed a new funding model. She's confident that it will help medical aids come to grips with longterm patient needs, and improve access to diagnosis and treatment. She believes that medical aid funding reluctance may be due to a lack of knowledge, and the risk of overdiagnosis - which further strengthens the case for raising the diagnostic bar.

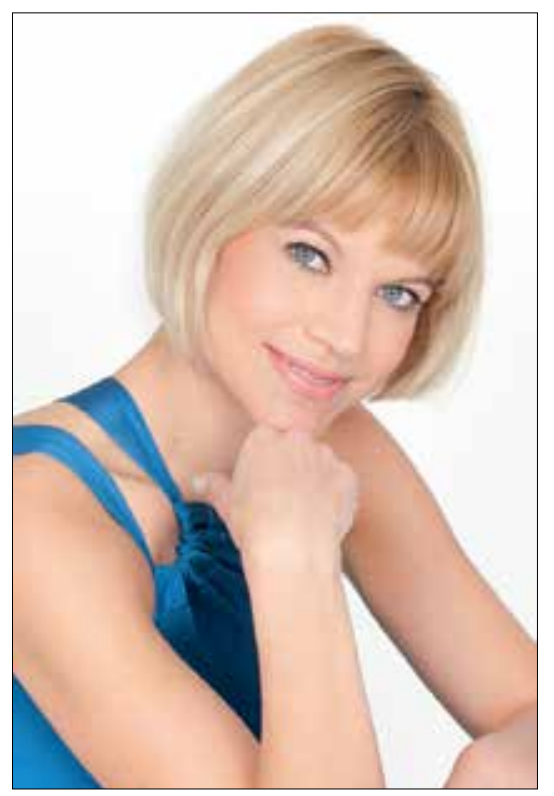

Dr Renata Schoeman, psychiatrist and top of her class at Stellenbosch University Business School.

Schoeman was judged SBC's top MBA student for 2015.

\section{Chris Bateman}

chrisb@hmpg.co.za

S Afr Med J 2016;106(5):431.

DOI:10.7196/SAMJ.2016.v106i5.10910 